\title{
Distributed State Estimation for Hybrid and Discrete Event Systems Using l-Complete Approximations *
}

\author{
Jörg Raisch ${ }^{*, * *}$ Thomas Moor $^{* *}$ Naim Bajcinca ${ }^{* * *}$ \\ Stephanie Geist* Vladislav Nenchev* \\ * Fachgebiet Regelungssysteme, Technische Universität Berlin, \\ Einsteinufer 17, 10587 Berlin, Germany (e-mail: \\ raisch@control.tu-berlin.de). \\ ** Lehrstuhl für Regelungstechnik, Universität Erlangen-Nürnberg, \\ Cauerstr. 7, 91058 Erlangen, Germany (e-mail: \\ thomas.moor@rt.eei.uni-erlangen.de) \\ *** Max-Planck-Institut für Dynamik komplexer technischer Systeme, \\ Sandtorstr. 1, 39108 Magdeburg, Germany
}

\begin{abstract}
The topic of this paper is distributed state estimation for time-invariant systems with finite input and output spaces. We assume that the system under investigation can be realised by a hybrid I/S/O-machine, where some of the discrete states may also represent failure modes. Our approach is based on previous work, e.g., Moor and Raisch (1999); Moor et al. (2002), where l-complete approximations were proposed as discrete event abstractions for hybrid dynamical systems. In particular, it has been shown that $l$-complete approximations can be used to provide set-valued estimates for the unknown system state. Estimates are conservative in the sense that the true state can be guaranteed to be contained in the set-valued estimate. In this contribution, we show that for a class of hybrid systems the same estimate can be obtained via a distributed, or decentralised, approach involving several less complex approximations, which are run in parallel. For a larger class of systems, it can be shown that this approach provides an outer approximation of the estimate provided by a monolithic $l$-complete estimator. The proposed procedure implies significant computational savings during estimator synthesis, with an only modest increase in on-line effort. The latter is a result of "assembling" the global estimate from the available local estimates. The resulting computational trade-off is explicitly discussed.
\end{abstract}

Keywords: Hybrid systems, discrete event systems, approximations, abstractions, behaviours, state estimation.

\section{INTRODUCTION}

In a series of previous papers, e.g., Moor and Raisch (1999); Moor et al. (2002), l-complete approximation has been suggested as a discrete event abstraction for a certain class of hybrid systems. This approach is based on Willems' behavioural systems theory, e.g., Willems (1989, 1991). For the purpose of controller design, two properties of $l$-complete approximation are important: (i) the behaviour (loosely speaking the set of external signals that is compatible with the system dynamics) of the approximation is guaranteed to contain the behaviour of the underlying hybrid system; (ii) for systems with finite external signal space, $l$-complete approximations can be realised by finite state machines. It can be shown (Moor and Raisch (1999)) that a particular intuitive realisation is past induced, i.e., at any instant of time, the state of the approximating automaton is uniquely determined by the past of the external signal. A straightforward modification

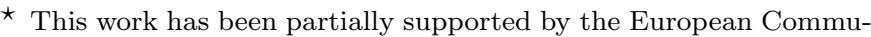
nity's Seventh Framework Programme under project DISC (GrantAgreement n. INFSO-ICT-224498).
}

turns this realisation into a set-valued estimator: each of the (finitely many) estimator states can be associated with a subset of the state space of the underlying hybrid system, and the subset associated with the current estimator state is guaranteed to contain the current state of the underlying hybrid system.

If some of the discrete states in the hybrid system model represent failure modes, the proposed distributed state estimation approach is also applicable for fault detection purposes.

It is a well-known fact that approximation accuracy is monotone in the parameter $l \in \mathbb{N}$. Correspondingly, the set-valued state estimates can be expected to "shrink" (and therefore to provide tighter bounds for the true state) if $l$ is increased. However, complexity of the resulting approximation automaton is exponential in $l$. This, in practice, often prevents the synthesis and use of a sufficiently accurate approximation. For control purposes, a number of extensions have been explored as possible antidotes, including modular (Moor et al. (2001)) and hierarchical control (Raisch and Moor (2005)). The present paper 
investigates how the complexity problem can be handled in an estimation context. It explores for a specific class of hybrid dynamical systems how a distributed approach can reduce complexity while maintaining estimation accuracy. It involves several $l$-complete approximations, each designed for a projection of the external signal space into an appropriate subspace, running in parallel and combining their local estimates to provide the desired global estimate. For a more general class of hybrid systems, the distributed estimate can still be shown to be an outer approximation of the set-valued estimate provided by a monolithic $l$ complete approximation.

Distributed state estimation for discrete event systems has been recognised as an important topic for quite some time, and a number of different approaches have been suggested to cover a variety of scenarios. Examples are Fabre et al. (2000); Xu and Kumar (2009); Jiroveanu et al. (2008).

This paper is organised as follows. In Section 2, we recall some basic concepts from Willems' behavioural systems theory. In Section 3, we summarize previous work on (strongest) $l$-complete approximations for hybrid systems. In Section 4, we briefly describe how an instantaneously observable realisation of such an approximation can serve as a set-valued estimator for the state of the underlying hybrid system. In Section 5, we address the distributed case. In particular, we investigate the case where the (finite) external signal space is the product of an input and an output space, and where each local estimator can only "see" the projection of the output signal into an appropriate subspace.

We use the following notation: capital letters denote signal spaces, e.g., $X, U, Y$, and $W$ represent the state space, the input and output spaces, and the external signal space, respectively. We work in discrete time, hence signals, which are denoted by minor letters, are sequences of symbols from the appropriate signal space, e.g., $w: \mathbb{N}_{0} \rightarrow W$ represents the external signal. The restriction of a signal to an in interval $[\tau, t], \tau, t \in \mathbb{N}_{0}, \tau \leq t$, is denoted by $\left.\cdot\right|_{[\tau, t]}$, e.g. $\left.w\right|_{[\tau, t]}=w(\tau), \ldots w(t)$. If appropriate, specific instances of a (restricted) signal are characterised by $\cdot$, e.g., $\left.\bar{w}\right|_{[0, t]}=\left\langle\omega_{0}, \ldots \omega_{t}\right\rangle$, with $\omega_{i} \in W$.

\section{SYSTEMS AND REALISATIONS}

Consider the hybrid state machine

$$
P=\left(X, W, \delta, X_{0}\right)
$$

where

$$
X=\mathbb{R}^{n} \times D, \quad|D|=m<\infty
$$

is the hybrid state space, $W$ is the finite external signal space, i.e., $|W|<\infty, \delta \subset X \times W \times X$ is the transition relation, and $X_{0} \subseteq X$ is the set of initial states. Clearly by setting $m=1$, we recover a purely continuous state space, while $n=0$ makes $P$ a finite state machine.

The full behaviour induced by $P$ is defined as (see Willems (1991))

$$
\begin{aligned}
& \mathfrak{B}_{s}:=\{(w, x) \mid(x(t), w(t),x(t+1)) \in \delta \\
&\left.\forall t \in \mathbb{N}_{0}, x(0) \in X_{0}\right\},
\end{aligned}
$$

where $x \in X^{\mathbb{N}_{0}}$ is a sequence of states (i.e., a state signal), and $w \in W^{\mathbb{N}_{0}}$ is a sequence of symbols from $W$ (i.e., an external signal). Hence the induced full behaviour consists of all pairs of state and external signals that are compatible with the state machine $P$.

$$
\begin{aligned}
\mathfrak{B} & :=\mathcal{P}_{W} \mathfrak{B}_{s} \\
& :=\left\{w \mid \exists x:(w, x) \in \mathfrak{B}_{s}\right\}
\end{aligned}
$$

is the canonical projection of $\mathfrak{B}_{s}$ onto the external external signal set and is referred to as the external behaviour induced by $P$. Following Willems (1989), we call

$$
\begin{aligned}
\Sigma_{s} & :=\left(\mathbb{N}_{0}, W, X, \mathfrak{B}_{s}\right) \\
\Sigma & :=\left(\mathbb{N}_{0}, W, \mathfrak{B}\right)
\end{aligned}
$$

the (state space) system induced by $P$, and we say that $P$ is a realisation of $\Sigma$, denoted by $P \cong \Sigma$.

Definition 1. See Willems (1991). A system $\Sigma=\left(\mathbb{N}_{0}, W, \mathfrak{B}\right)$ is said to be time invariant if $\sigma \mathfrak{B} \subseteq \mathfrak{B}$, where $\sigma$ is the backward shift operator, i.e., $\sigma w(t)=w(t+1)$ for all $t \in \mathbb{N}_{0}$.

Note that a sufficient (though not necessary) condition for $\Sigma$ to be time-invariant is that the initial conditions in a realisation $P \cong \Sigma$ are not restricted, i.e., $P=(X, W, \delta, X)$.

Definition 2. See Willems (1991). Let $l \in \mathbb{N}$. A time invariant dynamical system $\Sigma^{\prime}=\left(\mathbb{N}_{0}, W, \mathfrak{B}^{\prime}\right)$ is said to be l-complete if

$$
\left.\left.w \in \mathfrak{B}^{\prime} \Longleftrightarrow\left(\sigma^{t} w\right)\right|_{[0, l]} \in \mathfrak{B}^{\prime}\right|_{[0, l]} \quad \forall t \in \mathbb{N}_{0},
$$

where $\left.\cdot\right|_{[0, l]}$ denotes the restriction of a signal to the interval $[0, l]$.

\section{3. $L$-COMPLETE APPROXIMATIONS}

\subsection{The Concept of l-Complete Approximation}

We now recall the notion of (strongest) l-complete approximation, which was introduced in Moor and Raisch (1999). Roughly speaking, this is a system evolving on the same time axis $\mathbb{N}_{0}$ and within the same signal space $W$ as the original system, and with the smallest $l$-complete behaviour that covers the "original" behaviour $\mathfrak{B}$. Formally, this can be written as:

Definition 3. See Moor and Raisch (1999). Let $\Sigma=$ $\left(\mathbb{N}_{0}, W, \mathfrak{B}\right)$ and $\Sigma_{l}=\left(\mathbb{N}_{0}, W, \mathfrak{B}_{l}\right)$ be time invariant dynamical systems, with $l \in \mathbb{N}$. $\Sigma_{l}$ is said to be a strongest $l$-complete approximation induced by $\Sigma$ if the following conditions hold:

(i) $\mathfrak{B}_{l} \supseteq \mathfrak{B}, \mathfrak{B}_{l}$ is l-complete.

(ii) $\mathfrak{B}_{l}^{\prime} \supseteq \mathfrak{B}, \mathfrak{B}_{l}^{\prime}$ is $l$-complete $\quad \Longrightarrow \quad \mathfrak{B}_{l}^{\prime} \supseteq \mathfrak{B}_{l}$.

Proposition 1. (Moor and Raisch (1999)) Let $\Sigma=\left(\mathbb{N}_{0}, W\right.$, $\mathfrak{B})$ be a time invariant dynamical system. Choose an arbitrary $l \in \mathbb{N}$. Then, the strongest $l$-complete approximation induced by $\Sigma$, denoted by $\Sigma_{l}=\left(\mathbb{N}_{0}, W, \mathfrak{B}_{l}\right)$, exists uniquely, and $\mathfrak{B}_{l}$ is given by:

$$
\mathfrak{B}_{l}=\left\{\left.w\left|w \in W^{\mathbb{N}_{0}},\left(\sigma^{t} w\right)\right|_{[0, l]} \in \mathfrak{B}\right|_{[0, l]} \forall t \in \mathbb{N}_{0}\right\} .
$$

Note that, as $W$ is finite, $\left.\mathfrak{B}\right|_{[0, l]}$ is finite. Therefore, the strongest $l$-complete approximation can be realised by a finite state machine. Following Willems (1989), we can construct a suitable realisation by memorising the $l$ most 
recent external symbols. As our time axis is $\mathbb{N}_{0}$, we have to make provisions for the "start-up" phase, where less than $l$ external symbols are available. This is formalised in the following proposition.

Proposition 2. (Moor et al. (2002)) Let $\Sigma=\left(\mathbb{N}_{0}, W, \mathfrak{B}\right)$ be a time invariant system. Let

$$
Z_{l}:=\left\{\omega^{\star}\right\} \bigcup_{1 \leq r \leq l} W^{r}, \quad Z_{0}=\left\{\omega^{\star}\right\},
$$

where $\omega^{\star} \notin W$ is a new "dummy" symbol meaning "no external signal present so far". Let

$$
\delta_{l}:=\bigcup_{0 \leq r \leq l} \delta_{l}^{r} \subseteq Z_{l} \times W \times Z_{l},
$$

where

$$
\begin{aligned}
& \delta_{l}^{0}:=\left\{\left(\omega^{\star}, \omega_{0}, \omega_{0}\right)\left|\left\langle\omega_{0}\right\rangle \in \mathfrak{B}\right|_{[0,0]}\right\}, \\
& \delta_{l}^{r}:=\left\{\left(\left\langle\omega_{0}, \ldots \omega_{r-1}\right\rangle, \omega_{r},\left\langle\omega_{0}, \ldots \omega_{r}\right\rangle\right) \mid\right.\left.\left.\quad\left\langle\omega_{0}, \ldots \omega_{r}\right\rangle \in \mathfrak{B}\right|_{[0, r]}\right\}, \quad 1 \leq r<l, \\
& \delta_{l}^{l}:=\left\{\left(\left\langle\omega_{0}, \ldots \omega_{l-1}\right\rangle, \omega_{l},\left\langle\omega_{1}, \ldots \omega_{l}\right\rangle\right) \mid\right. \\
&\left.\left.\quad\left\langle\omega_{0}, \ldots \omega_{l}\right\rangle \in \mathfrak{B}\right|_{[0, l]}\right\} .
\end{aligned}
$$

Then $P_{l}:=\left(Z_{l}, W, \delta_{l}, Z_{0}\right)$ is a realisation of the strongest l-complete approximation $\Sigma_{l}$ of $\Sigma$.

To set up this realisation, we apparently have to decide whether strings $\left\langle\omega_{0}, \ldots \omega_{r}\right\rangle \in W^{r+1}$ are elements of the set $\left.\mathfrak{B}\right|_{[0, r]}, r \leq l$. This question will be answered in the next section for systems with a well defined input/output structure.

\section{2 l-Complete Approximations in an Input/Output Setting}

Definition 4. The hybrid state machine $P=\left(X, W, \delta, X_{0}\right)$ is called an I/S/O-machine, if

(i) $W=U \times Y$,

(ii) for every $\xi \in X$ and $\mu \in U$ there uniquely exist $\nu \in Y$ and $\xi^{\prime} \in X$ such that $\left(\xi,(\mu, \nu), \xi^{\prime}\right) \in \delta$.

In this case, $U$ and $Y$ are called the input and output space, respectively.

Clearly, item (ii) implies the existence of maps $f: X \times$ $U \rightarrow X$ and $g: X \times U \rightarrow Y$ such that

$$
\delta=\left\{\left(\xi,(\mu, \nu), \xi^{\prime}\right) \mid \xi^{\prime}=f(\xi, \mu), \nu=g(\xi, \mu)\right\} .
$$

As before, $P$ induces a system $\Sigma=\left(\mathbb{N}_{0}, U \times Y, \mathfrak{B}\right)$, with $\mathfrak{B} \subseteq(U \times Y)^{\mathbb{N}_{0}}$ and a state space system $\Sigma_{s}=$ $\left(\mathbb{N}_{0}, U \times \bar{Y}, X, \mathfrak{B}_{s}\right)$, with $\mathfrak{B}_{s} \subseteq(U \times Y \times X)^{\mathbb{N}_{0}}$. In Willems' terminology, $\Sigma$ is an I/O-system, while $\Sigma_{s}$ represents an $\mathrm{I} / \mathrm{S} / \mathrm{O}$-system.

Following Moor et al. (2002), we can now define the set of all states of an I/S/O-machine $P=(X, U \times Y, \delta, X)$ that are compatible with a given string $\left.(\bar{u}, \bar{y})\right|_{[0, r]}$ of input and output symbols:

$$
\begin{gathered}
\mathcal{X}\left(\left.(\bar{u}, \bar{y})\right|_{[0, r]}\right):= \\
\left\{\xi\left|\exists(u, y, x) \in \mathfrak{B}_{s}: x(r)=\xi,(u, y)\right|_{[0, r]}=\left.(\bar{u}, \bar{y})\right|_{[0, r]}\right\} .
\end{gathered}
$$

In particular, the string $\left.(\bar{u}, \bar{y})\right|_{[0, r]}$ occurs in the external behaviour if and only if the corresponding set of compatible state is non-empty, i.e.,

$$
\left.\left.(\bar{u}, \bar{y})\right|_{[0, r]} \in \mathfrak{B}\right|_{[0, r]} \Longleftrightarrow \mathcal{X}\left(\left.(\bar{u}, \bar{y})\right|_{[0, r]}\right) \neq \emptyset,
$$

and the finite state realisation $P_{l}$ of $\Sigma_{l}$ can therefore be derived by computing the sets $\mathcal{X}\left(\left.(\bar{u}, \bar{y})\right|_{[0, r]}\right)$. Note that, in general, $P_{l}$ will not be an I/S/O-machine.

As shown in Moor et al. (2002), the sets of compatible states can be characterized by the following iteration:

$$
\begin{aligned}
\mathcal{X}\left(\left.(\bar{u}, \bar{y})\right|_{[0,0]}\right) & =g_{\bar{u}(0)}^{-1}(\bar{y}(0)), \\
\mathcal{X}\left(\left.(\bar{u}, \bar{y})\right|_{[0, r+1]}\right) & =f\left(\mathcal{X}\left(\left.(\bar{u}, \bar{y})\right|_{[0, r]}\right), \bar{u}(r)\right) \\
& \cap g_{\bar{u}(r+1)}^{-1}(\bar{y}(r+1)),
\end{aligned}
$$

where $g_{\mu}^{-1}, \mu \in U$, denotes the inverse image of $g(\cdot, \mu)$, i. e. $g_{\mu}^{-1}(\nu):=\{\xi \mid g(\xi, \mu)=\nu\}$.

The computational techniques required to perform the above iteration depend on the particular class of hybrid state machine $P$ at hand, and, in general, build on a reachability analysis to establish images under $f$. This topic has been discussed intensively in the literature, for linear hybrid automata e.g. by Henzinger (1996), for switched linear systems e.g. by Chutinan and Krogh (1998), and for non-linear continuous dynamics by, e.g., Reißig (2009).

\section{SET-VALUED STATE ESTIMATION}

As pointed out in Moor and Raisch (1999), the realisation $P_{l}$ defined in Proposition 2 is past-induced, i.e., its present state $z_{l}(t) \in Z_{l}$ is uniquely determined by the past of the external signal, $\left.(u, y)\right|_{[0, t-1]}, t \in \mathbb{N}$. We now introduce a slightly modified state machine by defining

$$
\tilde{P}_{l}:=\left(\tilde{Z}_{l}, U \times Y, \tilde{\delta}_{l}, \tilde{Z}_{0}\right),
$$

with

$$
\begin{aligned}
\tilde{Z}_{l} & :=\bigcup_{1 \leq r \leq l}(U \times Y)^{r}, \\
\tilde{Z}_{l, 0} & :=U \times Y, \\
\tilde{\delta}_{l} & :=\bigcup_{1 \leq r \leq l} \tilde{\delta}_{l}^{r} \subseteq \tilde{Z}_{l} \times(U \times Y) \times \tilde{Z}_{l},
\end{aligned}
$$

where

$$
\begin{gathered}
\tilde{\delta}_{l}^{r}:=\left\{\left(\left\langle\left(\mu_{0}, \nu_{0}\right) \ldots\left(\mu_{r-1}, \nu_{r-1}\right)\right\rangle,\left(\mu_{r}, \nu_{r}\right),\left\langle\left(\mu_{0}, \nu_{0}\right), \ldots\right.\right.\right. \\
\left.\left.\left.\ldots\left(\mu_{r}, \nu_{r}\right)\right\rangle\right)\left|\left\langle\left(\mu_{0}, \nu_{0}\right), \ldots\left(\mu_{r}, \nu_{r}\right)\right\rangle \in \mathfrak{B}\right|_{[0, r]}\right\}, \\
1 \leq r<l \\
\tilde{\delta}_{l}^{l}:=\left\{\left(\left\langle\left(\mu_{0}, \nu_{0}\right) \ldots\left(\mu_{l-1}, \nu_{l-1}\right)\right\rangle,\left(\mu_{l}, \nu_{l}\right),\left\langle\left(\mu_{1}, \nu_{1}\right), \ldots\right.\right.\right. \\
\left.\left.\left.\ldots\left(\mu_{l}, \nu_{l}\right)\right\rangle\right)\left|\left\langle\left(\mu_{0}, \nu_{0}\right), \ldots\left(\mu_{l}, \nu_{l}\right)\right\rangle \in \mathfrak{B}\right|_{[0, l]}\right\} .
\end{gathered}
$$

Proposition 3. The state machines $P_{l}$ and $\tilde{P}_{l}$ induce the same external behaviour, i.e., $\tilde{P}_{l}$ is also a realisation of the strongest $l$-complete approximation $\Sigma_{l}$. Moreover, let $\mathfrak{B}_{l, s}$ and $\tilde{\mathfrak{B}}_{l, s}$ denote the full behaviours induced by $P_{l}$ and $\tilde{P}_{l}$, respectively. Then $\left(u, y, z_{l}\right) \in \mathfrak{B}_{l, s}$ if and only if $\left(u, y, \tilde{z}_{l}\right) \in \tilde{\mathfrak{B}}_{l, s}$ and $\tilde{z}_{l}=\sigma z_{l}$. 
Proof. The first part of the proof is a trivial extension of the proof in Moor et al. (2002). To show the second part, consider the temporal evolution of the states of $P_{l}$ and $\tilde{P}_{l}$ for an external signal $(\bar{u}, \bar{y})$. By construction, this is given by

$$
z_{l}(t)=\left\{\begin{array}{l}
\omega^{\star} \quad \text { if } t=0 \\
\left.(\bar{u}, \bar{y})\right|_{[0, t-1]} \quad \text { if } 0<t \leq l \\
\left.(\bar{u}, \bar{y})\right|_{[t-l, t-1]} \quad \text { if } t \geq l
\end{array}\right.
$$

and

$$
\tilde{z}_{l}(t)= \begin{cases}\left.(\bar{u}, \bar{y})\right|_{[0, t]} & \text { if } 0<t<l \\ \left.(\bar{u}, \bar{y})\right|_{[t-l+1, t]} \quad \text { if } t \geq l,\end{cases}
$$

respectively. Clearly, $\forall t \in \mathbb{N}, \tilde{z}_{l}(t)=z_{l}(t+1)$, i.e., $\tilde{z}=\sigma z$.

It is now straightforward to see that the realisation $\tilde{P}_{l}$ can serve as a current state estimator for the system $\Sigma_{s} \cong P($ Figure 1$)$ : the I/S/O-system $\Sigma_{s}=\left(\mathbb{N}_{0}, U \times\right.$ $\left.Y, X, \mathfrak{B}_{s}\right)$ is driven by the input signal $u$ and responds with an output $y$. Both signals serve as input for $\tilde{P}_{l}$. By construction, its current state $\tilde{z}_{l}(t)$ is uniquely determined by the present and the past of the external signal, i.e., $\left.(u, y)\right|_{[0, t]}$. Moreover, because of $(20)$, each element of the state set $\tilde{Z}_{l}$ is a string of pairs of input and output symbols and can therefore be uniquely associated with a set (16). This is the desired set-valued estimate for the current state of the system $\Sigma_{s}$.

Note that as an immediate consequence of the construction of the state estimator $\tilde{P}_{l}$, we have

$$
x(t) \in \mathcal{X}\left(\tilde{z}_{l}(t)\right) \forall t \in \mathbb{N}_{0}, \forall l \in \mathbb{N}
$$

and

$$
\mathcal{X}\left(\tilde{z}_{l+1}(t)\right) \subseteq \mathcal{X}\left(\tilde{z}_{l}(t)\right) \forall l \in \mathbb{N} .
$$

Hence, estimation is conservative in the sense that the estimate is guaranteed to contain the unknown state, and estimation accuracy is monotone in the parameter $l$. Unfortunately, increasing $l$ does not only refine the estimate, but complexity (e.g., $\left.\left|\tilde{Z}_{l}\right|\right)$ grows exponentially with $l$. This is the motivation for investigating distributed estimation schemes in the next section.

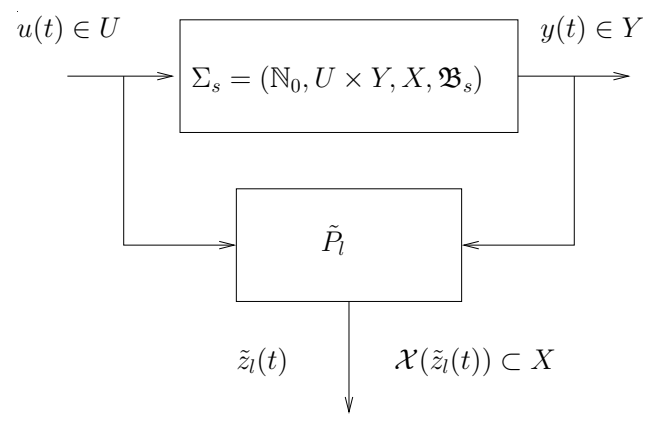

Fig. 1. Set-valued state estimation.

\section{DISTRIBUTED STATE ESTIMATION}

In the following, we assume that the output space can be decomposed as

$$
Y=Y^{(1)} \times \ldots \times Y^{(N)},
$$

hence $|Y|=\prod_{i=1}^{N}\left|Y^{(i)}\right|$. Furthermore, we assume that the map $f_{\mu}: X \rightarrow X$ defined by $f_{\mu}(\xi):=f(\xi, \mu)$ is injective for all $\mu \in U$. Note that is the case, e.g., when the system under investigation arises from sampling a switched locally Lipschitz-continuous autonomous vector field.

Let $\mathcal{P}^{(i)}$ denote the projection from $Y$ into $Y^{(i)}$ and, with slight abuse of notation, also from $Y^{\mathbb{N}_{0}}$ into $\left(Y^{(i)}\right)^{\mathbb{N}_{0}}$. We now design local estimators that can only "see" the input $u$ and the projection $y^{(i)}:=\mathcal{P}^{(i)} y, i=1, \ldots N$, with the aim to recover the "global" estimate $\mathcal{X}\left(\tilde{z}_{l}(t)\right)$ via the local estimators (see Fig. 2).

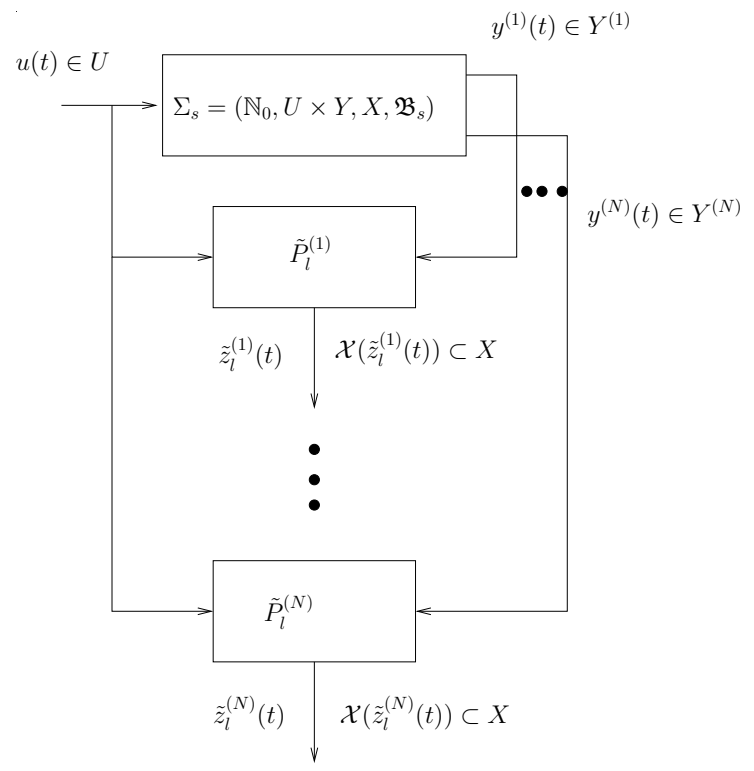

Fig. 2. Distributed set-valued state estimation.

The synthesis of each local estimator $\tilde{P}_{l}^{(i)}$ is based on the $\mathrm{I} / \mathrm{S} / \mathrm{O}-\mathrm{machine}$

$$
P^{(i)}:=\left(X, U \times Y^{(i)}, \delta^{(i)}, X\right)
$$

where the transition relation $\delta^{(i)}$ is defined by

$\left(\xi,\left(\mu, \nu^{(i)}\right), \xi^{\prime}\right) \in \delta^{(i)} \Longleftrightarrow \xi^{\prime}=f(\xi, \mu) \wedge \nu^{(i)}=\mathcal{P}^{(i)} g(\xi, \mu)$, and $f, g$ are the transition and output functions defining the transition relation of the $\mathrm{I} / \mathrm{S} / \mathrm{O}$-machine $P=(X, U \times$ $Y, \delta, X)$.

As discussed in the previous sections, each $P^{(i)}$ induces a time-invariant system $\Sigma^{(i)}=\left(\mathbb{N}_{0}, U \times Y^{(i)}, \mathfrak{B}^{(i)}\right)$ with unique strongest $l$-complete approximation $\Sigma_{l}^{(i)}=$ $\left(\mathbb{N}_{0}, U \times Y^{(i)}, \mathfrak{B}_{l}^{(i)}\right)$. Using the same procedure as in Section 4 , we now set up realisations $\tilde{P}_{l}^{(i)} \cong \Sigma_{l}^{(i)}, i=1, \ldots N$, and, as before, associate set-valued estimates with each element of the local estimator state sets $\tilde{Z}_{l}^{(i)}$.

Theorem 1. Consider a hybrid I/S/O-machine $P=(X, U \times$ $Y, \delta)$, with $\left(\xi,(\mu, \nu), \xi^{\prime}\right) \in \delta$ iff $\xi^{\prime}=f(\xi, \mu)$ and $\nu=g(\xi, \mu)$, where $f_{\mu}(\xi):=f(\xi, \mu)$ is injective for all $\mu \in U$. Assume that the output space can be decomposed as in (27). Then,

$$
\bigcap_{i=1}^{N} \mathcal{X}\left(\tilde{z}_{l}^{(i)}(t)\right)=\mathcal{X}\left(\tilde{z}_{l}(t)\right) \quad \forall t \in \mathbb{N}_{0},
$$

where $\tilde{z}_{l}^{(i)}(t)$ is the state of $\tilde{P}_{l}^{(i)}$ at time $t$, and $\mathcal{X}\left(\tilde{z}_{l}^{(i)}(t)\right)$ is the associated set-valued estimate. Hence, the estimate provided by the monolithic estimator $\tilde{P}_{l}$ can be exactly 
recovered by intersecting the local estimates provided by the machines $\tilde{P}_{l}^{(i)}, i=1, \ldots, N$.

Proof. Let $\bar{y}$ be the output response of system $\Sigma_{s}=$ $\left(\mathbb{N}_{0}, U \times Y, X, \mathfrak{B}_{s}\right)$ to an input signal $\bar{u}$. Then,

$$
\tilde{z}_{l}^{(i)}(t)=\left\{\begin{array}{lr}
\left.\left(\bar{u}, \mathcal{P}^{(i)} \bar{y}\right)\right|_{[0, t]} \quad \text { if } 0<t<l \\
\left.\left(\bar{u}, \mathcal{P}^{(i)} \bar{y}\right)\right|_{[t-l+1, t]} \quad \text { if } t \geq l
\end{array}\right.
$$

while $\tilde{z}_{l}(t)$ is given by $(24)$. Therefore

$$
\mathcal{X}\left(\tilde{z}_{l}^{(i)}(t)\right)=\left\{\begin{array}{l}
\mathcal{X}\left(\left.\left(\bar{u}, \mathcal{P}^{(i)} \bar{y}\right)\right|_{[0, t]}\right) \quad \text { if } 0<t<l \\
\mathcal{X}\left(\left.\left(\bar{u}, \mathcal{P}^{(i)} \bar{y}\right)\right|_{[t-l+1, t]}\right) \quad \text { if } t \geq l .
\end{array}\right.
$$

The proof is by induction. Clearly,

$$
\begin{aligned}
\bigcap_{i=1}^{N} \mathcal{X}\left(\tilde{z}_{l}^{(i)}(0)\right) & =\bigcap_{i=1}^{N}\left\{\xi \in X \mid \mathcal{P}^{(i)} g(\xi, \bar{u}(0))=\bar{y}^{(i)}(0)\right\} \\
& =\left\{\xi \in X \mid \bigwedge_{i=1}^{N}\left(\mathcal{P}^{(i)} g(\xi, \bar{u}(0))=\bar{y}^{(i)}(0)\right)\right\} \\
& =\{\xi \in X \mid g(\xi, \bar{u}(0))=\bar{y}(0)\} \\
& =\mathcal{X}\left(\tilde{z}_{l}(0)\right),
\end{aligned}
$$

i.e., (29) holds for $t=0$. Now assume that (29) holds for some $t \geq 0$. Then, for $t<l-1$, we can write

$$
\begin{aligned}
\bigcap_{i=1}^{N} \mathcal{X}\left(\tilde{z}_{l}^{(i)}(t+1)\right)= & \bigcap_{i=1}^{N} \mathcal{X}\left(\left(\left.\left(\bar{u}, \mathcal{P}^{(i)} \bar{y}\right)\right|_{[0, t+1]}\right)\right) \\
= & \bigcap_{i=1}^{N}\left(f\left(\mathcal{X}\left(\left.\left(\bar{u}, \mathcal{P}^{(i)} \bar{y}\right)\right|_{[0, t]}\right), \bar{u}(t)\right) \cap\right. \\
& \left.\left\{\xi \mid \mathcal{P}^{(i)} g(\xi, \bar{u}(t+1))=\bar{y}^{(i)}(t+1)\right\}\right) \\
= & \bigcap_{i=1}^{N} f\left(\mathcal{X}\left(\tilde{z}_{l}^{(i)}(t)\right), \bar{u}(t)\right) \\
& \bigcap_{i=1}^{N}\left\{\xi \mid \mathcal{P}^{(i)} g(\xi, \bar{u}(t+1))=\bar{y}^{(i)}(t+1)\right\} .
\end{aligned}
$$

Because $f_{\bar{u}(t)}(\cdot)=f(\cdot, \bar{u}(t))$ is injective for all $\bar{u}(t) \in U$, we have

$$
\begin{aligned}
\bigcap_{i=1}^{N} f\left(\mathcal{X}\left(\tilde{z}_{l}^{(i)}(t)\right), \bar{u}(t)\right) & =f\left(\left(\cap_{i=1}^{N} \mathcal{X}\left(\tilde{z}_{l}^{(i)}(t)\right)\right), \bar{u}(t)\right)(32) \\
& =f\left(\mathcal{X}\left(\tilde{z}_{l}(t)\right), \bar{u}(t)\right) \\
& =f\left(\mathcal{X}\left(\left.(\bar{u}, \bar{y})\right|_{[0, t]}, \bar{u}(t)\right) .\right.
\end{aligned}
$$

Furthermore,

$$
\begin{aligned}
\bigcap_{i=1}^{N}\{\xi \mid & \left.\mathcal{P}^{(i)} g(\xi, \bar{u}(t+1))=\bar{y}^{(i)}(t+1)\right\} \\
& =\left\{\xi \in X \mid \bigwedge_{i=1}^{N}\left(\mathcal{P}^{(i)} g(\xi, \bar{u}(t+1))=\bar{y}^{(i)}(t+1)\right)\right\} \\
& =\{\xi \mid g(\xi, \bar{u}(t+1))=\bar{y}(t+1)\} \\
& =g_{\bar{u}(t+1)}^{-1}(\bar{y}(t+1)),
\end{aligned}
$$

and therefore

$$
\begin{aligned}
\bigcap_{i=1}^{N} \mathcal{X}\left(\tilde{z}_{l}^{(i)}(t+1)\right) & =f\left(\mathcal{X}\left(\left.(\bar{u}, \bar{y})\right|_{[0, t]}, \bar{u}(t)\right)\right. \\
& \cap g_{\bar{u}(t+1)}^{-1}(\bar{y}(t+1)) \\
& =\mathcal{X}\left(\left.(\bar{u}, \bar{y})\right|_{[0, t+1]}\right) \\
& =\mathcal{X}\left(\tilde{z}_{l}(t+1)\right) .
\end{aligned}
$$

The proof for $t \geq l-1$ requires only slight modifications, but is given for completeness. In this case,

$$
\begin{aligned}
\bigcap_{i=1}^{N} \mathcal{X}\left(\tilde{z}_{l}^{(i)}(t+1)\right)= & \bigcap_{i=1}^{N} \mathcal{X}\left(\left(\left.\left(\bar{u}, \mathcal{P}^{(i)} \bar{y}\right)\right|_{[t-l+2, t+1]}\right)\right) \\
= & \bigcap_{i=1}^{N}\left(f\left(\mathcal{X}\left(\left.\left(\bar{u}, \mathcal{P}^{(i)} \bar{y}\right)\right|_{[t-l+2, t]}\right), \bar{u}(t)\right) \cap\right. \\
& \left.\left\{\xi \mid \mathcal{P}^{(i)} g(\xi, \bar{u}(t+1))=\bar{y}^{(i)}(t+1)\right\}\right) \\
= & \bigcap_{i=1}^{N} f\left(\mathcal{X}\left(\left.\left(\bar{u}, \mathcal{P}^{(i)} \bar{y}\right)\right|_{[t-l+2, t]}\right), \bar{u}(t)\right) \\
& \bigcap_{i=1}^{N}\left\{\xi \mid \mathcal{P}^{(i)} g(\xi, \bar{u}(t+1))=\bar{y}^{(i)}(t+1)\right\} .
\end{aligned}
$$

As $f_{\bar{u}(t)}(\cdot)=f(\cdot, \bar{u}(t))$ is injective for all $\bar{u}(t) \in U$, we have

$$
\begin{gathered}
\bigcap_{i=1}^{N} f\left(\mathcal{X}\left(\left.\left(\bar{u}, \mathcal{P}^{(i)} \bar{y}\right)\right|_{[t-l+2, t]}\right), \bar{u}(t)\right) \\
=f\left(\left(\cap_{i=1}^{N} \mathcal{X}\left(\left.\left(\bar{u}, \mathcal{P}^{(i)} \bar{y}\right)\right|_{[t-l+2, t]}\right)\right), \bar{u}(t)\right) \\
=f\left(\mathcal{X}\left(\left.(\bar{u}, \bar{y})\right|_{[t-l+2, t]}\right), \bar{u}(t)\right) .
\end{gathered}
$$

Because of (33), we get

$$
\begin{aligned}
\bigcap_{i=1}^{N} \mathcal{X}\left(\tilde{z}_{l}^{(i)}(t+1)\right) & =f\left(\mathcal{X}\left(\left.(\bar{u}, \bar{y})\right|_{[t-l+2, t]}, \bar{u}(t)\right)\right. \\
& \cap g_{\bar{u}(t+1)}^{-1}(\bar{y}(t+1)) \\
& =\mathcal{X}\left(\left.(\bar{u}, \bar{y})\right|_{[t-l+2, t+1]}\right) \\
& =\mathcal{X}\left(\tilde{z}_{l}(t+1)\right) .
\end{aligned}
$$

Remark: Note that the above proof relies crucially on the fact that $f_{\mu}(\cdot)=f(\cdot, \mu)$ is injective for all $\mu \in U$. This implies that $f(A, \mu) \cap f(B, \mu)=f(A \cap B, \mu)$ for arbitrary $A, B \subseteq X, \mu \in U$. For non-injective maps $f_{\mu}$, we still have $f(A, \mu) \cap f(B, \mu) \supseteq f(A \cap B, \mu)$ for arbitrary $A, B \subseteq X$, $\mu \in U$. This guarantees a weaker version of (29), stated in the following theorem.

Theorem 2. Consider a hybrid I/S/O-machine $P=(X, U \times$ $Y, \delta)$, with $\left(\xi,(\mu, \nu), \xi^{\prime}\right) \in \delta$ iff $\xi^{\prime}=f(\xi, \mu)$ and $\nu=g(\xi, \mu)$. Assume that the output space can be decomposed as in (27). Then,

$$
\bigcap_{i=1}^{N} \mathcal{X}\left(\tilde{z}_{l}^{(i)}(t)\right) \supseteq \mathcal{X}\left(\tilde{z}_{l}(t)\right) \quad \forall t \in \mathbb{N}_{0},
$$

where $\tilde{z}_{l}^{(i)}(t)$ is the state of $\tilde{P}_{l}^{(i)}, i=1, \ldots N$, at time $t$, and $\mathcal{X}\left(\tilde{z}_{l}^{(i)}(t)\right)$ is the associated set-valued estimate. Hence, intersecting the local estimates provides an outer 
approximation of $\mathcal{X}\left(\tilde{z}_{l}(t)\right)$ and is therefore guaranteed to contain the "true" state $x(t)$.

Proof. If $f_{\mu}(\cdot)=f(\cdot, \mu)$ is not injective, the "=" signs in (32) and (34) have to be replaced by " $\supseteq$ " signs. The claimed result then follows immediately.

We close with a brief discussion regarding possible reductions in complexity when replacing a monolithic $l$-complete estimator by the distributed scheme outlined above. In the monolithic case, the cardinality of the estimator state set is of the following order:

$$
\begin{aligned}
\left|\tilde{Z}_{l}\right| & \sim|U|^{l}|Y|^{l} \\
& \sim \mid U^{l}\left(\prod_{i=1}^{N}\left|Y^{(i)}\right|\right)^{l} .
\end{aligned}
$$

If $\left|Y^{(i)}\right| \leq p_{\max }, i=1, \ldots, N$,

$$
\left|\tilde{Z}_{l}\right| \sim|U|^{l} p_{\max }^{l N}
$$

i.e., the order of $\left|\tilde{Z}_{l}\right|$ is exponential in the number of components, $N$, of the output space $Y$. In contrast, the order of the cardinality of the state sets of the local estimators $\tilde{P}_{l}^{(i)}$ is

$$
\left|\tilde{Z}_{l}^{(i)}\right| \sim|U|^{l}\left|Y^{(i)}\right|^{l}, \quad i=1, \ldots, N .
$$

Hence, the overall number of estimator states in our distributed scheme is

$$
\sum_{i=1}^{N}\left|\tilde{Z}_{l}^{(i)}\right| \sim N|U|^{l} p_{\text {max }}^{l},
$$

i.e., complexity in the distributed case is linear in $N$. Hence, especially for large $N$, we may expect a very significant reduction in complexity when replacing the monolithic by a distributed estimation scheme. A similar argument applies to the computational effort needed to synthesise the monolithic estimator when compared to the distributed case. Note, however, that the reduction in estimator complexity comes at a price. Namely, the local estimators $\tilde{P}^{(i)}$ have to intersect their set-valued estimates on-line to provide the desired global estimate. The cost for this of course depends on the precise nature of the sets $\mathcal{X}\left(\tilde{z}_{l}^{(i)}(t)\right)$. For the well-known example of switched linear systems, these sets have the form of polytopes in $\mathbb{R}^{n}$, and the intersection operation can be efficiently implemented.

\section{CONCLUSION}

We have suggested a distributed set-valued estimation scheme for time-invariant dynamic systems that can be realised by hybrid I/S/O-machines with finite input and output spaces. The approach is based on the concept of l-complete approximations, e.g., Moor and Raisch (1999); Moor et al. (2002). For a suitable decomposition of the output space, we have proposed to replace the monolithic $l$-complete estimator by a set of "smaller" estimators running in parallel. Each of these local estimators is a realisation of an $l$-complete approximation of the projection of the system behaviour onto the appropriate component of the output space. We have shown that, in general, the resulting global estimate is an outer approximation of the estimation set provided by the monolithic estimator and therefore can be guaranteed to contain the actual system state. For a special case, characterised by an invertibility property of the transition function, we can show that the distributed approach exactly recovers the monolithic estimate. Such an invertibility assumption is of course restrictive, but holds for important classes of hybrid systems. An example are switched locally Lipschitz-continuous autonomous vector fields with finite input and output spaces. We have outlined that the distributed approach potentially implies a considerable reduction in estimator complexity. A further advantage of the suggested distributed approach, which still needs to be explored in detail, is a considerable increase in flexibility: in the monolithic case, estimation accuracy can only be improved "globally" by increasing the parameter $l$, which considerably increases complexity. In the distributed case, estimation accuracy can also be improved locally.

\section{REFERENCES}

Chutinan, A. and Krogh, B. (1998). Computing polyhedral approximations to flow pipes for dynamic systems. In Proc. 37th IEEE Conference on Decision and Control.

Fabre, E., Benveniste, A., Jard, C., Ricker, L.S., and Smith, M. (2000). Distributed state reconstruction for discrete event systems. In Proc. 39th IEEE Conference on Decision and Control, 2252-2257.

Henzinger, T. (1996). The theory of hybrid automata. In Proc. of 11th Annual IEEE Symposium on Logic in Computer Science (LICS'96), 278-292. IEEE Computer Society Press.

Jiroveanu, G., Boel, R., and Bordbar, B. (2008). Online monitoring of large petri net models under partial observation. Discrete Event Dynamic Systems, 18, 323354.

Moor, T., Davoren, J., and Raisch, J. (2001). Modular supervisory control of a class of hybrid systems in a behavioural framework. In Proceedings of the European Control Conference 2001, 870-875. Porto, Portugal.

Moor, T. and Raisch, J. (1999). Supervisory control of hybrid systems within a behavioural framework. Systems and Control Letters, 38, 157-166.

Moor, T., Raisch, J., and O'Young, S. (2002). Discrete supervisory control of hybrid systems based on $l$-complete approximations. Journal of Discrete Event Dynamic Systems, 12, 83-107.

Raisch, J. and Moor, T. (2005). Hierarchical hybrid control synthesis and its application to a multiproduct batch plant. In T. Meurer, K. Graichen, and E. Gilles (eds.), Control and Observer Design for Nonlinear Finite and Infinite Dimensional Systems, LNCIS 322, 199-216. Springer-Verlag.

Reißig, G. (2009). Computation of discrete abstractions of arbitrary memory span for nonlinear sampled systems. In R. Majumdar and P. Tabuada (eds.), Proc. 12th Intl. Conf. Hybrid Systems: Computation and Control (HSCC), San Francisco, U.S.A., April 13-15, 2009, volume 5469 of Lect. Notes Computer Science, 306-320.

Willems, J. (1989). Models for dynamics. Dynamics Reported, 2, 172-269.

Willems, J. (1991). Paradigms and puzzles in the theory of dynamic systems. IEEE Transactions on Automatic Control, 36, 258-294.

$\mathrm{Xu}$, S. and Kumar, R. (2009). Distributed state estimation in discrete event systems. In Proc. 2009 American Control Conference. 